\title{
Control of Hyperglycemia Using Differentiated and Undifferentiated Mesenchymal Stem Cells in Rats with Type 1 Diabetes
}

\author{
Ehsan Aali ${ }^{\mathrm{a}}$, Zahra Madjd $^{\mathrm{b}}$, Neda Tekiyehmaroof ${ }^{\mathrm{c}}$, Ali Mohammad Sharifi $^{\mathrm{c}}$
}

${ }^{a}$ Department of Pharmacology, Qazvin University of Medical Sciences, Qazvin, Iran

${ }^{b}$ Department of Pathology, Oncology Research Center, Iran University of Medical Sciences, Tehran, Iran

${ }^{\mathrm{c}}$ Razi Drug Research Center, Department of Pharmacology, School of Medicine, Iran University of Medical Sciences, Hemmat Highway, Tehran, 1449614535, Iran

\begin{abstract}
Due to their ability in self-renewing and differentiation into a wide variety of tissues, mesenchymal stem cells (MSCs) exhibit outstanding potential for regenerative medicine. This study was aimed at investigating different aspects of MSC therapy in controlling hyperglycemia in streptozotocin-induced diabetes rats. Using an islet cell differentiation protocol, bone marrow (BM) MSCs were differentiated into insulin-producing cells (IPCs). The differentiation process was evaluated by immunocytochemistry, reverse transcriptase PCR, and dithizone staining. Diabetic animals in 4 diabetic individual groups received normal saline, BM-MSCs, coadministration of BM-MSCs with supernatant, and IPCs. Blood glucose and insulin levels were monitored during the experiment. Immunohistochemical analysis of the pancreas was performed at the end of the experiment. Administration of BM-MSCs could not reverse glucose and insulin levels in experimental animals as efficiently as cotransplantation of BM-MSCs with supernatant. The effect of coadministration of BM-MSCs with supernatant and transplantation of IPCs on controlling hyperglycemia is comparable. Immunohistochemical analysis showed that number and size of islets per section were significantly increased in groups receiving IPCs and BM-MSC-supernatant compared to the MSC group of animals. In conclusion, coadministration of BM-MSCs with supernatant could be used as efficiently as IPC transplantation in controlling hyperglycemia in diabetic rats.
\end{abstract}

\section{Author keywords}

Coadministration; Diabetes mellitus; Insulinproducing cells; Mesenchymal stem cell; Transplantation 